\title{
Nutrient intake of European adolescents: results of the HELENA (Healthy Lifestyle in Europe by Nutrition in Adolescence) Study
}

\author{
Katharina Diethelm ${ }^{1}$ * , Inge Huybrechts ${ }^{2}$, Luis Moreno ${ }^{3}$, Stefaan De Henauw ${ }^{2,4}$, \\ Yannis Manios 5 , Laurent Beghin 6,7, Marcela González-Gross ${ }^{8}$, Cinzia Le Donne 9 \\ Magdalena Cuenca-García ${ }^{10}$, Manuel J Castillo ${ }^{10}$, Kurt Widhalm ${ }^{11}$, \\ Emma Patterson ${ }^{12,13}$ and Mathilde Kersting ${ }^{1}$ \\ ${ }^{1}$ Research Institute of Child Nutrition, Rheinische Friedrich-Wilhelms-University Bonn, Heinstueck 11, 44225 \\ Dortmund, Germany: ${ }^{2}$ Department of Public Health, Faculty of Medicine and Health Sciences, Ghent University, \\ Ghent, Belgium: ${ }^{3}$ Escuela Universitaria de Ciencias de la Salud, Universidad de Zaragoza, Zaragoza, Spain: \\ ${ }^{4}$ Department of Health Sciences, Vesalius Hogeschool Gent, Ghent, Belgium: ${ }^{5}$ Department of Nutrition and \\ Dietetics, Harokopio University, Athens, Greece: ${ }^{6} \mathrm{C}$ entre d'Investigation Clinique de Lille, Lille, France: ${ }^{7}$ Unité \\ Inserm U995 \& Université Lille Nord de France, Lille, France: ${ }^{8}$ ImFINE Research Group, Department of Health \\ and Human Performance, Faculty of Physical Activity and Sport Sciences - INEF, Technical University of Madrid, \\ Madrid, Spain: ${ }^{9}$ National Research Institute on Food and Nutrition, Rome, Italy: ${ }^{10}$ Department of Medical \\ Physiology, School of Medicine, University of Granada, Granada, Spain: ${ }^{11}$ Academic Institute for Clinical \\ Nutrition, Vienna, Austria: ${ }^{12}$ Unit for Preventive Nutrition, Department of Biosciences and Nutrition, Karolinska \\ Institute, Stockholm, Sweden: ${ }^{13}$ Division of Social Medicine, Department of Public Health Sciences, Karolinska \\ Institute, Stockholm, Sweden
}

Submitted 13 August 2012: Final revision received 9 January 2013: Accepted 22 January 2013: First published online 8 March 2013

\begin{abstract}
Objective: An adequate nutritional intake in childhood and adolescence is crucial for growth and the prevention of youth and adult obesity and nutrition-related morbidities. Improving nutrient intake in children and adolescents is of public health importance. The purpose of the present study was to describe and evaluate the nutrient intake in a European sample using the D-A-CH nutrient intake recommendations and the Nutritional Quality Index (NQI).

Design: The HELENA (Healthy Lifestyle in Europe by Nutrition in Adolescence) Study is a cross-sectional study, the main objective of which is to obtain comparable data on a variety of nutritional and health-related parameters in adolescents aged $12 \cdot 5-17 \cdot 5$ years.

Setting: Eight cities in Europe.

Subjects: The initial sample consisted of 3528 European adolescents. Among these, 1590 adolescents (54\% female) had sufficient and plausible dietary data on energy and nutrient intakes from two $24 \mathrm{~h}$ recalls using the HELENA-DIAT software.

Results: The intakes of most macronutrients, vitamins and minerals were in line with the D-A-CH recommendations. While the intakes of SFA and salt were too high, the intake of PUFA was too low. Furthermore, the intakes of vitamin D, folate, iodine and $\mathrm{F}$ were less than about $55 \%$ of the recommendations. The median NQI was about 71 (of a maximum of 100).

Conclusions: The intakes of most nutrients were adequate. However, further studies using suitable criteria to assess nutrient status are needed. Public health initiatives should educate children and adolescents regarding balanced food choices.
\end{abstract}

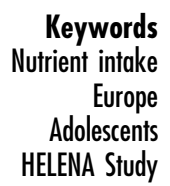

There is recent evidence that adequate nutritional intake by children and adolescents is associated with a lower risk of youth ${ }^{(1,2)}$ and adult obesity ${ }^{(3)}$ as well as of subsequent nutrition-related morbidities in adulthood such as $\mathrm{CVD}^{(4,5)}$, several cancers ${ }^{(6,7)}$ and type 2 diabetes $^{(8,9)}$.

Adolescents experience pubertal hormonal changes regulating appetite, satiety and body fat distribution ${ }^{(10)}$ and increasingly assert independence from their parents, all of which may affect their eating behaviour ${ }^{(11)}$. Thus adolescence is a critical period $^{(12,13)}$ in which poor dietary practices may constitute an increased risk for several adult chronic diseases $^{(8)}$. Furthermore, dietary habits which have been established in childhood or adolescence may track into adult life ${ }^{(14)}$. Improving the nutritional intake of children and adolescents is therefore a goal for global public health $^{(15,16)}$. 
Several surveys have been conducted on the nutritional intake of European adolescents; however, they have mostly been conducted on national, regional or in nonrepresentative samples ${ }^{(17-20)}$. For instance, compared with nutrient intake recommendations, the average intake of sucrose was higher and the average intakes of fibre, Fe, folate and vitamin D were lower in 14-year-old Finnish adolescents $^{(17)}$. German adolescents aged 11-19 years had a slightly higher proportion of energy intake from fat, while the intakes of micronutrients were in line with the recommendations, except for folate, $\mathrm{Fe}$ and $\mathrm{Ca}$ in girls $^{(18)}$. In 10- to 18-year-old Italian teenagers and 6- to 7-year-old Spanish pupils, the proportion of energy from fat was higher than recommended and the proportion from carbohydrates was lower ${ }^{(20,21)}$. Both the Italian and the Spanish adolescents met the recommended intakes for micronutrients ${ }^{(20,21)}$.

However, these surveys differ in methodology, population groups and age categories, and thus the available information is insufficient for a comprehensive conclusion about the nutritional status of adolescents at a pan-European level ${ }^{(22)}$. The Healthy Lifestyle in Europe by Nutrition in Adolescence (HELENA) Cross-Sectional Study (HELENA-CSS) gathered, for the first time, representative data about the health and nutritional status of a large sample of adolescents across European countries assessed with standardized, harmonized and validated instruments and procedures.

The purpose of the present analysis was to describe and evaluate the nutritional intake by means of the recommendations of the nutrition societies of Germany, Austria and Switzerland (D-A-CH) ${ }^{(23)}$, which are based on a public health-oriented concept and set recommended intakes at a level intended to prevent nutrient-specific deficiency symptoms, but also achieve optimal nutrition and avoid oversupply with energy or certain nutrients ${ }^{(24)}$. In a further step, the nutrient intake data of the European adolescents were condensed to a single parameter, the Nutritional Quality Index (NQI), to assess overall diet quality ${ }^{(25)}$. The NQI was constructed on the basis of nutritional recommendations as evaluation criteria ${ }^{(26)}$ and was used as a summary measure of the degree to which the adolescents' diet conformed to the D-A-CH recommendations.

\section{Methods}

\section{Study design and sample}

The HELENA-CSS was conducted between 2006 and 2007 in ten European cities (Athens, Heraklion (Greece), Dortmund (Germany), Ghent (Belgium), Lille (France), Pecs (Hungary), Rome (Italy), Stockholm (Sweden), Vienna (Austria) and Zaragoza (Spain)). Detailed information about the study has been reported elsewhere ${ }^{(27,28)}$. The main objective of HELENA-CSS was to obtain reliable and comparable data on a variety of nutritional and health-related parameters in a representative sample of 3528 European adolescents (boys and girls aged $12 \cdot 5-17 \cdot 5$ years $)^{(28,29)}$. The ethical committee of each centre approved the study and signed informed consent was obtained from the adolescents as well as from the parents $^{(30)}$.

For the present analysis, data on nutritional intake from Heraklion and Pecs ( $n$ 678) could not be included because of incomplete data. Furthermore, specific inclusion criteria (complete energy and nutrient intake data on two days obtained by the HELENA-DIAT $24 \mathrm{~h}$ recall and data on anthropometry) were defined for the present analysis, which were fulfilled by 1804 adolescents. Finally, adolescents with plausible dietary recalls were ascertained by relating their reported total energy intake to their BMR, as described below. Hence, the cohort analysed here included 1590 adolescents (54\% female).

\section{Dietary intake assessment}

Dietary intake data were obtained using a self-administered, computerized $24 \mathrm{~h}$ recall, named HELENA-DIAT, which was based on the Young Adolescents' Nutrition Assessment on Computer (YANA-C) ${ }^{(31)}$, a tool validated in Flemish adolescents. The basic version was improved and culturally adapted by adding national dishes to reach a European standard ${ }^{(32)}$. The dietary data collection is organized in six meal occasions, i.e. breakfast, morning snack, lunch, afternoon snack, evening meal and evening snack. The participants can select from about 400 predefined food items and are free to add non-listed foods manually. Special techniques are used to allow a detailed description and quantification of foods, e.g. pictures of portion sizes and dishes. Amounts eaten could be reported as grams or by common household measures. After a short introduction by a trained researcher, the adolescents completed the HELENA-DIAT $24 \mathrm{~h}$ recall during school time while a research staff member was present in the classroom to assist the adolescents if necessary. They completed the HELENA-DIAT twice on non-consecutive days within a time span of 2 weeks, to achieve information closer to habitual food intake than assessing food intake on consecutive days. The two $24 \mathrm{~h}$ recalls thus comprised weekdays and weekend days, but not necessarily a weekday and weekend day for each individual.

To calculate energy and nutrient intake, data of the HELENA-DIAT were linked to the German Food Code and Nutrient Database (BLS (Bundeslebensmittelschlüssel) version $\mathrm{II} \cdot 3 \cdot 1,2005)^{(33)}$. For this purpose, culture-specific composite dishes were disaggregated into their basic food components, all of which were available in the German database. In addition to energy, a total of thirty-two nutrients (macronutrients, vitamins and minerals) were assessed. The Multiple Source Method was used to adjust the intake data taking into account both betweenand within-individual variability of the dietary intake ${ }^{(34)}$. However, table salt, fortified foods and supplement consumption were not assessed in HELENA-DIAT. 
The daily intakes of each nutrient as well as total daily energy intake were calculated for each participant from the mean of the two $24 \mathrm{~h}$ recalls. Total daily energy intake was used to exclude potentially implausible recalls by comparing it with BMR estimated using the equations of Schofield ${ }^{(35)}$. Using the approach of Goldberg et al. ${ }^{(36)}$, fifty-one ( $47 \%$ male and 53\% female) adolescents were considered as under-reporters (ratio of energy intake to BMR <0.99) and 163 (61\% male and 39\% female) adolescents were considered as over-reporters (ratio of energy intake to BMR rate $>2 \cdot 40$ ) and were therefore excluded from the analysis.

The purpose of the present data analysis was to comprehensively describe the nutritional intake of European adolescents and to evaluate it in the light of recommended intakes. We used the age- and sex-specific reference values for nutrient intake provided by the nutrition societies of Germany, Austria and Switzerland $(\mathrm{D}-\mathrm{A}-\mathrm{CH})^{(23)}$, since these recommendations provide data for all thirty-two nutrients in HELENA ${ }^{(24)}$ and are used in two of the eight HELENA cities. The individual percentage achievement of the recommendations for the particular nutrients was ascertained, since the D-A-CH values do not include a specific value to compare mean intakes of groups with the recommended value.

In order to assess the overall health-related quality of dietary habits as a summary measure, Gedrich and Karg developed the Nutritional Quality Index (NQI) ${ }^{(26)}$. For this analysis we calculated two approaches of the NQI, one based on absolute values and one based on nutrient densities. The NQI is based on the proportional intake as compared with the reference values. In order to calculate the NQI, the nutrients are classified into two groups: (i) those which should not exceed the recommended level; and (ii) those which should not fall below the recommended level. Intakes that meet the corresponding reference values are rated with an intake quality score (IQS) of 100. For nutrients of the first group the exceeding portion is subtracted from 100 to form the IQS, e.g. SFA intake of $130 \%$ of the recommendation results in an IQS of 70 . For the second group the portion that is below the recommendation is subtracted from 100 , e.g. a vitamin C intake of $80 \%$ of the recommendation results in an IQS of 80. In a further step, the NQI is calculated as the harmonic mean of all IQS values for each individual. Thus, the NQI scores range from 0 to 100 , with a higher score indicating better diet quality ${ }^{(26)}$.

\section{Antbropometric measurements}

Measurements were performed by trained staff in a standardized way ${ }^{(37)}$, with the adolescents barefoot and in underwear. Weight was measured with an electronic scale to the nearest $0 \cdot 1 \mathrm{~kg}$, and height was measured with a telescopic stadiometer to the nearest $0 \cdot 1 \mathrm{~cm}$. BMI was calculated from height and weight $\left(\mathrm{kg} / \mathrm{m}^{2}\right)$. Sex- and ageindependent BMI standard deviation scores (SDS) were calculated using $Z$-values for BMI, calculated via the LMS method by Cole et al. ${ }^{(38)}$. Overweight was defined according to age- and sex-standardized BMI cut-off points based on data of Cole et al. ${ }^{(38)}$. Skinfold thicknesses were measured three times on the left side of the body with a Holtain calliper to the nearest $0 \cdot 2 \mathrm{~mm}$. Body fat percentage $(\mathrm{BF} \%)$ was calculated from triceps and subscapular skinfold thicknesses using Slaughter et al.'s equations ${ }^{(39)}$.

\section{Socio-economic characteristics}

Socio-economic characteristics were assessed with a selfreported questionnaire ${ }^{(40)}$. The adolescents reported whether their parents were overweight (yes/no) and their parents' educational level (lower education/lower secondary or higher secondary/higher education/university degree) ${ }^{(41)}$. The familial affluence scale (FAS), previously validated ${ }^{(42)}$, was used as an indicator of the adolescents' material affluence. It was based on information about family car ownership, having an own bedroom, Internet availability and computer ownership. Furthermore, migration status (born outside the country in which they lived during the study: yes/no) was assessed.

\section{Health-related characteristics}

Physical activity was assessed with the self-administered International Physical Activity Questionnaire for Adolescents (IPAQ-A) covering questions about physical activity during the last $7 \mathrm{~d}^{(43)}$. The IPAQ-A validity was tested by comparing its results with accelerometer data ${ }^{(44)}$. Total minutes per week (min/week) were computed and assigned to moderate-to-vigorous physical activity according to the guidelines for data processing ${ }^{(45)}$. Additionally, smoking status (ever smoked: yes/no) was assessed.

\section{Nutritional knowledge characteristics}

To assess the nutritional knowledge of the adolescents, a validated nutritional knowledge test was used ${ }^{(46)}$. For evaluation, the percentage of correct answers to the twenty-three multiple-choice questions was calculated.

\section{Statistical analysis}

SAS procedures (SAS statistical software package version 9.13) were used for data analysis. A $P$ value $<0 \cdot 05$ was considered statistically significant. Sample weights were applied to adjust for exclusion of study participants due to insufficient data. The sampling weight calibrates the sample so that it matches the theoretical sample with regard to sex and age group.

Differences in characteristics between included and excluded participants and NQI values between boys and girls were tested using ANOVA for normally distributed variables, Kruskal-Wallis tests for non-normally distributed variables and $\chi^{2}$ tests for categorical variables. Values are presented as weighted frequencies, means and standard deviations, or medians with 25 th and 75 th percentiles. 
Weighted intakes of nutrients by the adolescents were calculated as medians (25th and 75 th percentiles). The percentage intake of the reference value was calculated and presented as medians (25th and 75th percentiles) of the respective percentages of each individual's achievement compared with the D-A-CH recommendations.

\section{Results}

The present data analysis included 731 (46\%) boys and 859 (54\%) girls. Characteristics regarding anthropometry, socio-economic status, health status and nutritional knowledge for the included and excluded participants are presented in Table 1. The included had a lower BMISDS, percentage of overweight and $\mathrm{BF} \%$ (all $P<0 \cdot 0001$ ). Differences between the included and excluded regarding socio-economic characteristics were also statistically significant ( $P$ values $<0 \cdot 0001$ or $<0 \cdot 0005$ ) except for migration status, although percentages of parental overweight and education status as well as familial affluence were comparable. The included furthermore reported significantly less to have ever smoked $(P<0 \cdot 0001)$ and significantly less physical activity $(P=0 \cdot 02)$ compared with the excluded.

The weighted dietary intakes of nutrients and energy of the European boys and girls, stratified by age group, compared with the recommendations are shown in Tables 2 and 3. The fulfilment of the recommendations for both genders is additionally illustrated in Fig. 1. The recommendations for daily nutrient intake were designed to meet the nutrient requirements of nearly all healthy individuals $^{(47)}$, i.e. the recommended values are about $20-30 \%$ above the average requirement ${ }^{(23)}$. Thus, we considered an intake of $75-125 \%$ of the recommended values as acceptable.

Regarding protein, the intake was about twice as high as the recommendation, i.e. about 200 and $170 \%$ of the nutrient reference value in boys and girls of all age groups, respectively. While cholesterol intake and fat intake in general were approximately in line with the recommendations in both genders, the intake of SFA was about $40 \%$ higher than recommended, whereas the intake of PUFA was about $40 \%$ lower than the nutrient reference value. Intake of carbohydrates was in line with the recommendations in both genders and all age groups and also fibre intake was within the acceptable range of the reference values with a slightly higher intake among girls compared with boys.

The fat-soluble vitamins $\mathrm{A}$ and $\mathrm{E}$ were found to be within the acceptable range, with the exception of vitamin $\mathrm{E}$ in boys of the two younger age groups whose intake was only about $70 \%$ of the recommendation.

Table 1 Characteristicst of the study sample: 1590 included and 1938 excluded European adolescents from the Healthy Lifestyle in Europe by Nutrition in Adolescents (HELENA) study

\begin{tabular}{|c|c|c|c|c|c|c|c|}
\hline \multirow[b]{3}{*}{ Variables } & \multicolumn{6}{|c|}{ HELENA participants } & \multirow[b]{3}{*}{$\begin{array}{c}P \text { for } \\
\text { difference }\end{array}$} \\
\hline & \multicolumn{3}{|c|}{ Included ( $n$ 1590) } & \multicolumn{3}{|c|}{ Excluded ( $n$ 1938) } & \\
\hline & $\begin{array}{l}\text { Median or } \\
\text { Mean }\end{array}$ & $\begin{array}{l}\text { P25, P75 } \\
\text { or SD }\end{array}$ & $n$ & $\begin{array}{l}\text { Median or } \\
\text { Mean }\end{array}$ & $\begin{array}{l}\text { P25, P75 } \\
\text { or SD }\end{array}$ & $n$ & \\
\hline Age (years) & $14 \cdot 7$ & $13 \cdot 7,15 \cdot 7$ & 1590 & $14 \cdot 7$ & $13 \cdot 7,15 \cdot 8$ & 1938 & $>0.9$ \\
\hline \multicolumn{8}{|l|}{ Anthropometric characteristics } \\
\hline BMI-SDS§ & $0 \cdot 3$ & $1 \cdot 1$ & 1590 & $0 \cdot 7$ & $1 \cdot 1$ & 1938 & $<0.0001$ \\
\hline Overweight (\%)\| & \multicolumn{2}{|c|}{$16 \cdot 4$} & 1590 & \multicolumn{2}{|c|}{$28 \cdot 7$} & 1938 & $<0.0001$ \\
\hline $\mathrm{BF} \%$ & $21 \cdot 6$ & $16 \cdot 2,27 \cdot 1$ & 1554 & 23.9 & $17 \cdot 0,30 \cdot 7$ & 1781 & $<0.0001$ \\
\hline \multicolumn{8}{|l|}{ Socio-economic characteristics } \\
\hline Maternal overweight (\%)+† & \multicolumn{2}{|c|}{$10 \cdot 6$} & 1590 & \multicolumn{2}{|c|}{$12 \cdot 2$} & 1938 & $<0.0001$ \\
\hline Paternal overweight (\%)+t & \multicolumn{2}{|c|}{$15 \cdot 8$} & 1590 & \multicolumn{2}{|c|}{$14 \cdot 0$} & 1938 & $<0.0001$ \\
\hline High maternal education (\%)ł‡ & \multicolumn{2}{|c|}{$68 \cdot 6$} & 1519 & \multicolumn{2}{|c|}{$62 \cdot 0$} & 1796 & $<0.0001$ \\
\hline 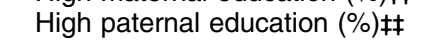 & \multirow{2}{*}{\multicolumn{2}{|c|}{$65 \cdot 5$}} & 1476 & \multicolumn{2}{|c|}{$59 \cdot 4$} & 1726 & $<0.0005$ \\
\hline High familial affluence (\%) $\$ \S$ & & & 1590 & \multicolumn{2}{|c|}{$26 \cdot 2$} & 1938 & $<0.0001$ \\
\hline Migrant (\%)|||| & \multicolumn{2}{|c|}{$\begin{array}{r}33.5 \\
7.8\end{array}$} & 1568 & \multicolumn{2}{|c|}{$6 \cdot 6$} & 1856 & 0.2 \\
\hline \multicolumn{6}{|l|}{ Health-related characteristics } & & \\
\hline Ever smoked (\%) & \multicolumn{2}{|c|}{34.9} & 1590 & \multicolumn{2}{|c|}{$40 \cdot 5$} & 1938 & $<0.0001$ \\
\hline Physical activity (min/week) & 540 & 270,995 & 1495 & 600 & 270,1115 & 1546 & 0.02 \\
\hline Nutritional knowledgettt & $60 \cdot 9$ & $47 \cdot 8,69 \cdot 6$ & 1567 & $60 \cdot 9$ & $47 \cdot 8,69 \cdot 6$ & 1858 & 0.9 \\
\hline
\end{tabular}

P25, 25th percentile; P75, 75th percentile; SDS, standard deviation score; BF\%, body fat percentage.

+Values are presented as weighted median with P25 and P75, mean and standard deviation, or frequency.

$\ddagger$ Significant differences between groups tested using the Kruskal-Wallis test for non-normally distributed variables, ANOVA for normally distributed variables and the $\chi^{2}$ test for categorical variables.

§BMI-SDS calculated using Z-values for BMI, calculated via the LMS method by Cole et al. ${ }^{(38)}$

$\|$ Derived from the sex- and age-specific cut-offs proposed by the International Obesity Taskforce, which correspond to an adult BMI cut-off of $25 \mathrm{~kg} / \mathrm{m}^{2(38)}$

Estimated according to the equations of Slaughter et al. ${ }^{(39)}$

t+Obtained from questions about adolescents' perception.

¥¥Higher secondary education and higher education or university degree.

$\S \S$ Based on information about family car ownership, having an own bedroom, Internet availability and computer ownership.

III Participant was born outside the country in which they lived during the study.

$\uparrow \uparrow$ Moderate-to-vigorous physical activity.

t+tPercentage of correctly answered questions in the nutritional knowledge test. 
Table 2 Weighted dietary nutrient and energy intakes in comparison with the D-A-CH reference values for nutrient intake, stratified by age group, of 731 European boys aged $12 \cdot 5-17 \cdot 5$ years from the Healthy Lifestyle in Europe by Nutrition in Adolescents (HELENA) Study

\begin{tabular}{|c|c|c|c|c|c|c|c|c|c|c|c|c|c|c|c|}
\hline \multirow[b]{4}{*}{ Nutrient } & \multicolumn{15}{|c|}{ Age (years)t } \\
\hline & \multicolumn{5}{|c|}{10 to $<13(n 48)$} & \multicolumn{5}{|c|}{13 to $<15(n 365)$} & \multicolumn{5}{|c|}{15 to $<19(n 318)$} \\
\hline & \multicolumn{2}{|r|}{ Intake } & \multirow[b]{2}{*}{ Ref. value $\ddagger$} & \multicolumn{2}{|c|}{$\%$ intake of ref. value $\S$} & \multicolumn{2}{|c|}{ Intake } & \multirow[b]{2}{*}{ Ref. value $\ddagger$} & \multicolumn{2}{|c|}{$\%$ intake of ref. value $\S$} & \multicolumn{2}{|c|}{ Intake } & \multirow[b]{2}{*}{ Ref. value $\ddagger$} & \multicolumn{2}{|c|}{$\%$ intake of ref. value $\S$} \\
\hline & Median & P25, P75 & & Median & P25, P75 & Median & P25, P75 & & Median & P25, P75 & Median & P25, P75 & & Median & P25, P75 \\
\hline Protein $(\mathrm{g} / \mathrm{kg})$ & $2 \cdot 1$ & $1 \cdot 6,2 \cdot 4$ & 0.9 & 228 & 177,264 & $1 \cdot 8$ & $1 \cdot 5,2 \cdot 2$ & 0.9 & 205 & 166,248 & $1 \cdot 7$ & $1 \cdot 3,1 \cdot 9$ & 0.9 & 185 & 149,217 \\
\hline Fat $(\% \mathrm{E})$ & $33 \cdot 4$ & $29 \cdot 6,37 \cdot 0$ & 30 & 111 & 99,123 & $33 \cdot 6$ & $30 \cdot 3,36 \cdot 6$ & 30 & 112 & 101,122 & $34 \cdot 3$ & $31 \cdot 3,37 \cdot 9$ & 30 & 114 & 104,126 \\
\hline SFA (\%E) & $14 \cdot 0$ & $11 \cdot 3,15 \cdot 4$ & $<10$ & 140 & 113,154 & $14 \cdot 1$ & $12 \cdot 5,15 \cdot 7$ & $<10$ & 141 & 125,157 & $14 \cdot 4$ & $12 \cdot 6,15 \cdot 9$ & $<10$ & 144 & 126,159 \\
\hline MUFA (\%E) & $12 \cdot 5$ & $11 \cdot 0,13 \cdot 9$ & 10 & 125 & 110,139 & $12 \cdot 2$ & $10 \cdot 9,13 \cdot 7$ & 10 & 122 & 109,137 & $12 \cdot 6$ & $11 \cdot 2,14 \cdot 0$ & 10 & 126 & 112,140 \\
\hline PUFA (\%E) & $4 \cdot 2$ & $3 \cdot 8,5 \cdot 2$ & 10 & 61 & 54,75 & $4 \cdot 2$ & $3 \cdot 7,4 \cdot 9$ & 10 & 59 & 52,70 & $4 \cdot 5$ & $3 \cdot 8,5 \cdot 5$ & 10 & 65 & 55,79 \\
\hline Cholesterol (mg) & 352 & 284,448 & 300 & 117 & 95,149 & 380 & 310,457 & 300 & 127 & 103,152 & 412 & 337,490 & 300 & 137 & 112,163 \\
\hline Carbohydrates (\%E) & $49 \cdot 0$ & $44 \cdot 5,54 \cdot 9$ & $>50$ & 98 & 89,110 & $49 \cdot 8$ & $45 \cdot 8,53 \cdot 5$ & $>50$ & 100 & 92,107 & $48 \cdot 9$ & $45 \cdot 5,53 \cdot 2$ & $>50$ & 98 & 91,106 \\
\hline Fibre $(g / 4184 \mathrm{~kJ}(1000 \mathrm{kcal}))$ & $7 \cdot 9$ & $6 \cdot 7,9 \cdot 1$ & 10 & 80 & 70,93 & $7 \cdot 9$ & $6 \cdot 7,9 \cdot 0$ & 10 & 80 & 68,91 & $7 \cdot 3$ & $6 \cdot 5,8 \cdot 6$ & 10 & 75 & 66,88 \\
\hline Vitamin A (mg) & 0.9 & $0 \cdot 7,1 \cdot 2$ & 0.9 & 100 & 80,132 & $1 \cdot 0$ & $0 \cdot 8,1 \cdot 3$ & $1 \cdot 1$ & 92 & 74,116 & $1 \cdot 1$ & $0 \cdot 9,1 \cdot 3$ & $1 \cdot 1$ & 101 & 78,120 \\
\hline Vitamin D ( $\mu \mathrm{g})$ & 1.8 & $1 \cdot 3,2 \cdot 3$ & 5 & 36 & 27,46 & $2 \cdot 0$ & $1 \cdot 5,2 \cdot 6$ & 5 & 40 & 31,52 & $2 \cdot 1$ & $1 \cdot 6,2 \cdot 8$ & 5 & 42 & 31,56 \\
\hline Vitamin E (mg) & $9 \cdot 1$ & $7 \cdot 7,11 \cdot 1$ & 13 & 70 & 59,85 & $9 \cdot 5$ & $8 \cdot 1,11 \cdot 4$ & 14 & 68 & 58,82 & $11 \cdot 3$ & $9 \cdot 5,13 \cdot 4$ & 15 & 76 & 63,90 \\
\hline Vitamin K ( $\mu \mathrm{g})$ & 223 & 175,289 & 40 & 557 & 437,723 & 226 & 189,281 & 50 & 453 & 378,562 & 247 & 203,306 & 70 & 353 & 290,437 \\
\hline Thiamin (mg) & $1 \cdot 3$ & $1 \cdot 2,1 \cdot 5$ & $1 \cdot 2$ & 111 & 97,128 & $1 \cdot 3$ & $1 \cdot 1,1 \cdot 6$ & $1 \cdot 4$ & 96 & 81,114 & 1.5 & $1 \cdot 2,1 \cdot 8$ & $1 \cdot 3$ & 115 & 95,135 \\
\hline Riboflavin (mg) & $1 \cdot 7$ & $1 \cdot 3,2 \cdot 0$ & $1 \cdot 4$ & 124 & 94,142 & $1 \cdot 8$ & $1 \cdot 5,2 \cdot 1$ & $1 \cdot 6$ & 111 & 92,132 & $1 \cdot 7$ & $1 \cdot 4,2 \cdot 1$ & $1 \cdot 5$ & 114 & 96,140 \\
\hline Niacin $(m g)$ & $15 \cdot 9$ & $13 \cdot 5,19 \cdot 4$ & 15 & 106 & 90,129 & $15 \cdot 9$ & $13 \cdot 0,19 \cdot 4$ & 18 & 88 & 72,108 & $17 \cdot 0$ & $14 \cdot 1,20 \cdot 0$ & 17 & 100 & 83,118 \\
\hline Vitamin $B_{6}(\mathrm{mg})$ & $1 \cdot 7$ & $1 \cdot 5,2 \cdot 1$ & $1 \cdot 0$ & 173 & 145,211 & $1 \cdot 8$ & $1 \cdot 5,2 \cdot 2$ & $1 \cdot 4$ & 130 & 107,155 & 1.9 & $1 \cdot 6,2 \cdot 3$ & 1.6 & 118 & 99,142 \\
\hline Folate $(\mu \mathrm{g})$ & 202 & 175,249 & 400 & 50 & 44,62 & 217 & 178,258 & 400 & 54 & 45,64 & 221 & 186,271 & 400 & 55 & 46,68 \\
\hline Pantothenic acid (mg) & $5 \cdot 2$ & $4 \cdot 4,6 \cdot 2$ & 6 & 104 & 87,124 & $5 \cdot 4$ & $4 \cdot 5,6 \cdot 3$ & 6 & 89 & 75,105 & $5 \cdot 4$ & $4 \cdot 5,6 \cdot 4$ & 6 & 90 & 75,106 \\
\hline Biotin $(\mu \mathrm{g})$ & $45 \cdot 4$ & $34 \cdot 1,49 \cdot 8$ & 20 & 227 & 170,249 & $46 \cdot 3$ & $38 \cdot 8,54 \cdot 7$ & 25 & 185 & 155,219 & $47 \cdot 2$ & $38 \cdot 8,54 \cdot 9$ & 30 & 158 & 129,183 \\
\hline Vitamin $B_{12}(\mu \mathrm{g})$ & $6 \cdot 6$ & $4 \cdot 6,8 \cdot 1$ & $2 \cdot 0$ & 328 & 231,407 & $6 \cdot 3$ & $5 \cdot 0,7 \cdot 6$ & $3 \cdot 0$ & 211 & 165,255 & $6 \cdot 2$ & $4 \cdot 9,7 \cdot 6$ & $3 \cdot 0$ & 207 & 164,254 \\
\hline Vitamin C (mg) & $90 \cdot 6$ & $51 \cdot 3,127 \cdot 4$ & 90 & 101 & 57,142 & $90 \cdot 4$ & $61 \cdot 4,127 \cdot 7$ & 100 & 90 & 61,128 & $90 \cdot 1$ & $64 \cdot 4,131 \cdot 7$ & 100 & 90 & 64,132 \\
\hline $\mathrm{Na}(\mathrm{mg}) \|$ & 2170 & 1943,2741 & $510^{\circ}$ & 425 & 381,537 & 2540 & 2093,3055 & 550 ฯ & 462 & 381,556 & 2761 & 2332, 3284 & 550 ฯ & 502 & 424,597 \\
\hline $\mathrm{Cl}(\mathrm{mg}) \|$ & 3776 & 3340,4426 & $770^{\circ}$ & 490 & 434,575 & 4313 & 3694,5008 & $830^{\circ}$ & 520 & 445,603 & 4578 & 4016,5216 & $830^{\circ}$ & 551 & 484,628 \\
\hline $\mathrm{K}(\mathrm{mg}) \|$ & 2797 & 2257,3111 & $1700^{\circ}$ & 165 & 133,183 & 2954 & 2515,3501 & $1900 \uparrow$ & 155 & 132, 184 & 3008 & 2532,3584 & $2000 \pi$ & 150 & 127,179 \\
\hline $\mathrm{Ca}(\mathrm{mg})$ & 859 & 695,1046 & 1100 & 78 & 63,95 & 937 & 662,1229 & 1200 & 78 & 55,102 & 857 & 561,1130 & 1200 & 72 & 47,94 \\
\hline$P(\mathrm{mg})$ & 1427 & 1219,1618 & 1250 & 114 & 98,129 & 1532 & 1301,1784 & 1250 & 123 & 104,143 & 1572 & 1320,1815 & 1250 & 126 & 106,145 \\
\hline $\mathrm{Mg}(\mathrm{mg})$ & 315 & 279,368 & 230 & 137 & 121,160 & 349 & 298,403 & 310 & 112 & 96,130 & 361 & 309,424 & 400 & 90 & 77,106 \\
\hline $\mathrm{Fe}(\mathrm{mg})$ & $12 \cdot 8$ & $11 \cdot 7,14 \cdot 9$ & 12 & 107 & 97,124 & 13.9 & $11 \cdot 9,16 \cdot 1$ & 12 & 116 & 99,134 & $14 \cdot 7$ & $13 \cdot 1,17 \cdot 0$ & 10 & 123 & 109,142 \\
\hline lodine $(\mu \mathrm{g})$ & $97 \cdot 7$ & $82 \cdot 8,119 \cdot 1$ & 180 & 54 & 46,66 & 108 & $89 \cdot 8,127 \cdot 9$ & 200 & 54 & 45,64 & 109 & $91 \cdot 7,127 \cdot 7$ & 200 & 54 & 46,64 \\
\hline $\mathrm{F}(\mathrm{mg})$ & 0.6 & $0 \cdot 5,0 \cdot 7$ & $2 \cdot 0$ & 30 & 27,36 & 0.7 & $0 \cdot 6,0 \cdot 8$ & $3 \cdot 2$ & 22 & 19,25 & 0.8 & $0 \cdot 7,0.9$ & $3 \cdot 2$ & 24 & 20,28 \\
\hline $\mathrm{Zn}(\mathrm{mg})$ & $12 \cdot 1$ & $11 \cdot 3,13 \cdot 9$ & $9 \cdot 0$ & 135 & 126,154 & $13 \cdot 1$ & $11 \cdot 4,15 \cdot 1$ & $9 \cdot 5$ & 138 & 120,159 & $13 \cdot 9$ & $12 \cdot 0,15 \cdot 7$ & $10 \cdot 0$ & 139 & 120,157 \\
\hline $\mathrm{Cu}(\mathrm{mg})$ & 1.9 & $1 \cdot 7,2 \cdot 4$ & $1 \cdot 0$ & 194 & 175,240 & $2 \cdot 2$ & $1 \cdot 9,2 \cdot 5$ & $1 \cdot 0$ & 218 & 186,253 & $2 \cdot 3$ & $2 \cdot 0,2 \cdot 7$ & $1 \cdot 0$ & 233 & 202,267 \\
\hline Energy (MJ) & $9 \cdot 44$ & $8 \cdot 70,10 \cdot 64$ & 8.45 & 111 & 103,126 & $10 \cdot 54$ & $9 \cdot 41,1.93$ & 9.96 & 106 & 95,120 & $11 \cdot 74$ & $10 \cdot 32,13 \cdot 15$ & $11 \cdot 42$ & 109 & 96,122 \\
\hline Energy (kcal) & 2255 & 2080,2544 & 2020 & 111 & 103,126 & 2518 & 2249,2852 & 2380 & 106 & 95,120 & 2806 & 2465,3144 & 2730 & 109 & 96,122 \\
\hline
\end{tabular}

P25, 25th percentile; P75, 75th percentile; \%E, percentage of energy intake.

tValues are presented as median with P25 and P75.

¥D-A-CH reference values for nutrient intake of the German Nutrition Society, Austrian Nutrition Society, Swiss Society for Nutrition Research and Swiss Nutrition Association ${ }^{(23)}$.

\$Median (P25, P75) of the respective percentages of each individual's intake compared with the D-A-CH reference values.

IIDiscretionary salt intake was not included.

- Estimated values for minimum intake. 
Table 3 Weighted dietary nutrient and energy intakes in comparison with the D-A-CH reference values for nutrient intake, stratified by age group, of 859 European girls aged $12 \cdot 5-17 \cdot 5$ years from the Healthy Lifestyle in Europe by Nutrition in Adolescents (HELENA) Study

\begin{tabular}{|c|c|c|c|c|c|c|c|c|c|c|c|c|c|c|c|}
\hline \multirow[b]{4}{*}{ Nutrient } & \multicolumn{15}{|c|}{ Age (years) +} \\
\hline & \multicolumn{5}{|c|}{10 to $<13(n 51)$} & \multicolumn{5}{|c|}{13 to $<15$ ( $n 451)$} & \multicolumn{5}{|c|}{15 to $<19$ ( $n 357)$} \\
\hline & \multicolumn{2}{|c|}{ Intake } & \multirow[b]{2}{*}{ Ref. value $\ddagger$} & \multicolumn{2}{|c|}{$\%$ intake of ref. value } & \multicolumn{2}{|c|}{ Intake } & \multirow[b]{2}{*}{ Ref. value $\ddagger$} & \multicolumn{2}{|c|}{$\%$ intake of ref. value $\S$} & \multicolumn{2}{|c|}{ Intake } & \multirow[b]{2}{*}{ Ref. value $\ddagger$} & \multicolumn{2}{|c|}{$\%$ intake of ref. value } \\
\hline & Median & P25, P75 & & Median & P25, P75 & Median & P25, P75 & & Median & P25, P75 & Median & P25, P75 & & Median & P25, P75 \\
\hline Protein $(\mathrm{g} / \mathrm{kg})$ & 1.5 & $1 \cdot 4,1 \cdot 8$ & 0.9 & 169 & 152, 202 & 1.5 & $1 \cdot 3,1 \cdot 8$ & 0.9 & 166 & 142, 201 & $1 \cdot 4$ & $1 \cdot 2,1 \cdot 6$ & 0.8 & 178 & 146, 205 \\
\hline Fat $(\% \mathrm{E})$ & 34.0 & $31 \cdot 1,38 \cdot 4$ & 30 & 113 & 104,128 & $34 \cdot 0$ & $30 \cdot 5,37 \cdot 6$ & 30 & 113 & 102,125 & 34.5 & $31 \cdot 1,37 \cdot 5$ & 30 & 115 & 104,125 \\
\hline SFA (\%E) & $14 \cdot 4$ & $12 \cdot 8,16 \cdot 5$ & $<10$ & 144 & 128,165 & $14 \cdot 0$ & $12 \cdot 5,15 \cdot 7$ & $<10$ & 140 & 125,157 & $14 \cdot 1$ & $12 \cdot 5,15 \cdot 6$ & $<10$ & 141 & 125,156 \\
\hline MUFA (\%E) & $12 \cdot 7$ & $10 \cdot 9,14 \cdot 5$ & 10 & 127 & 109,145 & $12 \cdot 3$ & $10 \cdot 9,13 \cdot 9$ & 10 & 123 & 109,139 & $12 \cdot 7$ & $11 \cdot 2,13 \cdot 8$ & 10 & 127 & 112,138 \\
\hline PUFA (\%E) & $4 \cdot 6$ & $4 \cdot 0,5 \cdot 1$ & 10 & 66 & 57,73 & $4 \cdot 7$ & $4 \cdot 0,5 \cdot 5$ & 10 & 66 & 57,78 & $4 \cdot 8$ & $4 \cdot 1,5 \cdot 6$ & 10 & 69 & 59,80 \\
\hline Cholesterol (mg) & 302 & 252,371 & 300 & 101 & 84,124 & 310 & 264,371 & 300 & 103 & 88,124 & 309 & 260,366 & 300 & 103 & 87,122 \\
\hline Carbohydrates (\%E) & $48 \cdot 7$ & $44 \cdot 1,53 \cdot 4$ & $>50$ & 97 & 88,107 & $49 \cdot 8$ & $46 \cdot 0,53 \cdot 9$ & $>50$ & 100 & 92,108 & $49 \cdot 6$ & $45 \cdot 9,53 \cdot 4$ & $>50$ & 99 & 92,107 \\
\hline Fibre $(\mathrm{g} / 4184 \mathrm{~kJ}(1000 \mathrm{kcal}))$ & $7 \cdot 9$ & $7 \cdot 2,9 \cdot 0$ & 10 & 80 & 73,91 & $8 \cdot 6$ & $7 \cdot 6,10 \cdot 2$ & 10 & 87 & 77,103 & $8 \cdot 8$ & $7 \cdot 5,10 \cdot 1$ & 10 & 89 & 76,102 \\
\hline Vitamin A (mg) & 0.9 & $0.7,1 \cdot 1$ & 0.9 & 95 & 82,127 & 0.9 & $0 \cdot 8,1 \cdot 1$ & $1 \cdot 0$ & 92 & 78,114 & $1 \cdot 0$ & $0 \cdot 8,1 \cdot 2$ & 0.9 & 106 & 89,132 \\
\hline Vitamin D $(\mu \mathrm{g})$ & $1 \cdot 6$ & $1 \cdot 3,2 \cdot 2$ & 5 & 33 & 26,44 & $1 \cdot 8$ & $1 \cdot 4,2 \cdot 2$ & 5 & 36 & 28,44 & 1.8 & $1 \cdot 5,2 \cdot 2$ & 5 & 36 & 29,45 \\
\hline Vitamin E (mg) & 8.6 & $7 \cdot 1,9 \cdot 4$ & 13 & 79 & 65,86 & 8.9 & $7 \cdot 5,10 \cdot 3$ & 14 & 74 & 63,86 & $9 \cdot 5$ & $7 \cdot 9,11 \cdot 1$ & 15 & 79 & 66,93 \\
\hline Vitamin $\mathrm{K}(\mu \mathrm{g})$ & 200 & 167,232 & 40 & 499 & 417,580 & 207 & 173,252 & 50 & 413 & 346,504 & 208 & 177,252 & 60 & 347 & 296,420 \\
\hline Thiamin (mg) & $1 \cdot 1$ & $0.9,1.3$ & $1 \cdot 0$ & 108 & 88,126 & $1 \cdot 1$ & $0.9,1 \cdot 3$ & $1 \cdot 1$ & 99 & 86,115 & $1 \cdot 1$ & $1 \cdot 0,1 \cdot 3$ & $1 \cdot 0$ & 111 & 95,128 \\
\hline Riboflavin (mg) & $1 \cdot 4$ & $1 \cdot 2,1 \cdot 6$ & $1 \cdot 2$ & 115 & 102,131 & $1 \cdot 4$ & $1 \cdot 2,1 \cdot 6$ & $1 \cdot 3$ & 105 & 90,122 & 1.3 & $1 \cdot 1,1 \cdot 6$ & $1 \cdot 2$ & 112 & 94,132 \\
\hline Niacin (mg) & $12 \cdot 3$ & $11 \cdot 2,15 \cdot 4$ & 13 & 95 & 86,119 & $13 \cdot 0$ & $11 \cdot 2,15 \cdot 7$ & 15 & 87 & 74,104 & $13 \cdot 1$ & $10 \cdot 9,15 \cdot 8$ & 13 & 101 & 84,122 \\
\hline Vitamin $B_{6}(\mathrm{mg})$ & $1 \cdot 4$ & $1 \cdot 3,1 \cdot 9$ & 1.0 & 144 & 128,188 & 1.5 & $1 \cdot 3,1 \cdot 8$ & 1.4 & 108 & 93,127 & 1.5 & $1 \cdot 2,1 \cdot 8$ & $1 \cdot 2$ & 123 & 104,146 \\
\hline Folate $(\mu \mathrm{g})$ & 177 & 147,213 & 400 & 44 & 37,53 & 187 & 162,217 & 400 & 47 & 40,54 & 195 & 160,225 & 400 & 49 & 40,56 \\
\hline Pantothenic acid (mg) & $4 \cdot 2$ & $3 \cdot 7,4 \cdot 8$ & 6 & 84 & 74,96 & $4 \cdot 3$ & $3 \cdot 8,5 \cdot 0$ & 6 & 72 & 63,83 & $4 \cdot 3$ & $3 \cdot 6,5 \cdot 1$ & 6 & 72 & 61,84 \\
\hline Biotin $(\mu \mathrm{g})$ & 38.7 & $32 \cdot 3,44 \cdot 2$ & 20 & 193 & 162,221 & $37 \cdot 8$ & $33 \cdot 3,44 \cdot 7$ & 25 & 151 & 133,179 & $37 \cdot 2$ & $31 \cdot 8,44 \cdot 8$ & 30 & 124 & 106,149 \\
\hline Vitamin $B_{12}(\mu \mathrm{g})$ & $4 \cdot 7$ & $4 \cdot 1,6 \cdot 0$ & $2 \cdot 0$ & 236 & 204,298 & 4.7 & $4 \cdot 0,5 \cdot 9$ & $3 \cdot 0$ & 156 & 133,195 & 4.7 & $3 \cdot 8,5 \cdot 9$ & 3.0 & 157 & 128,198 \\
\hline Vitamin C (mg) & $77 \cdot 0$ & $54 \cdot 3,132 \cdot 1$ & 90 & 86 & 60,147 & $91 \cdot 0$ & $64 \cdot 0,132 \cdot 3$ & 100 & 91 & 64,132 & $86 \cdot 8$ & $60 \cdot 3,124 \cdot 7$ & 100 & 87 & 60,125 \\
\hline $\mathrm{Na}(\mathrm{mg}) \|$ & 1965 & 1563,2393 & $510^{\circ}$ & 385 & 307,469 & 2012 & 1681,2463 & 550 ฯ & 366 & 306,448 & 2072 & 1791,2488 & $550 \%$ & 377 & 326,452 \\
\hline $\mathrm{Cl}(\mathrm{mg}) \|$ & 3269 & 2774,3963 & $770^{\circ}$ & 425 & 360,515 & 3373 & 2924, 3951 & $830^{\circ}$ & 406 & 352,476 & 3516 & 3072,4042 & $830^{\circ}$ & 424 & 370,487 \\
\hline $\mathrm{K}(\mathrm{mg}) \|$ & 2451 & 20142921 & $1700^{\circ}$ & 144 & 118,172 & 2491 & 2146,2920 & $1900^{\star}$ & 131 & 113,154 & 2475 & 2093,2936 & $2000^{\star}$ & 124 & 105,147 \\
\hline $\mathrm{Ca}(\mathrm{mg})$ & 809 & 635,953 & 1100 & 74 & 58,87 & 727 & 483,955 & 1200 & 61 & 40,80 & 661 & 459,880 & 1200 & 55 & 38,73 \\
\hline$P(\mathrm{mg})$ & 1197 & 1053,1297 & 1250 & 96 & 84,104 & 1220 & 1063,1400 & 1250 & 98 & 85,112 & 1212 & 1039,1440 & 1250 & 97 & 83,115 \\
\hline $\mathrm{Mg}(\mathrm{mg})$ & 278 & 240,305 & 250 & 111 & 96,122 & 291 & 251,330 & 310 & 94 & 81,107 & 294 & 249,346 & 350 & 84 & 71,99 \\
\hline $\mathrm{Fe}(\mathrm{mg})$ & $10 \cdot 9$ & $9 \cdot 3,12 \cdot 6$ & 15 & 73 & 62,84 & $11 \cdot 2$ & $10 \cdot 1,12 \cdot 9$ & 15 & 75 & 67,86 & 11.8 & $10 \cdot 2,13 \cdot 5$ & 15 & 79 & 68,89 \\
\hline lodine $(\mu \mathrm{g})$ & $92 \cdot 1$ & $84 \cdot 0,104 \cdot 4$ & 180 & 51 & 47,58 & 92.8 & $79 \cdot 2,108 \cdot 5$ & 200 & 46 & 40,54 & 90.5 & $77 \cdot 2,107 \cdot 8$ & 200 & 45 & 39,54 \\
\hline $\mathrm{F}(\mathrm{mg})$ & 0.6 & $0.5,0.7$ & $2 \cdot 0$ & 28 & 23,34 & 0.6 & $0.5,0 \cdot 7$ & 2.9 & 20 & 17,24 & 0.6 & $0.5,0.7$ & $2 \cdot 9$ & 22 & 18,26 \\
\hline $\mathrm{Zn}(\mathrm{mg})$ & $10 \cdot 2$ & $9 \cdot 1,11 \cdot 4$ & 7.0 & 145 & 130,163 & 10.5 & $9 \cdot 2,11 \cdot 9$ & $7 \cdot 0$ & 150 & 131,170 & $10 \cdot 8$ & $9 \cdot 2,12 \cdot 3$ & 7.0 & 154 & 131,176 \\
\hline $\mathrm{Cu}(\mathrm{mg})$ & 1.9 & $1 \cdot 6,2 \cdot 0$ & 1.0 & 186 & 162,203 & 1.9 & $1 \cdot 6,2 \cdot 1$ & 1.0 & 187 & 164,211 & 1.9 & $1 \cdot 7,2 \cdot 2$ & 1.0 & 194 & 168,224 \\
\hline Energy (MJ) & $8 \cdot 46$ & $7 \cdot 40,9 \cdot 29$ & $7 \cdot 36$ & 115 & 101,126 & 8.52 & $7 \cdot 65,9 \cdot 62$ & $8 \cdot 12$ & 105 & 94,119 & 8.75 & $7 \cdot 90,9 \cdot 77$ & $9 \cdot 20$ & 101 & 91,113 \\
\hline Energy (kcal) & 2021 & 1769,2220 & 1760 & 115 & 101,126 & 2036 & 1828,2300 & 1940 & 105 & 94,119 & 2091 & 1889,2336 & 2200 & 101 & 91,113 \\
\hline
\end{tabular}

P25, 25th percentile; P75, 75th percentile; \%E, percentage of energy intake.

†D-A-CH reference values for nutrient intake of the German Nutrition Society, Austrian Nutrition Society, Swiss Society for Nutrition Research and Swiss Nutrition Association ${ }^{(23)}$.

\$Median (P25, P75) of the respective percentages of each individual's intake compared with the D-A-CH reference values.

IDiscretionary salt intake was not included.

- Estimated values for minimum intake. 


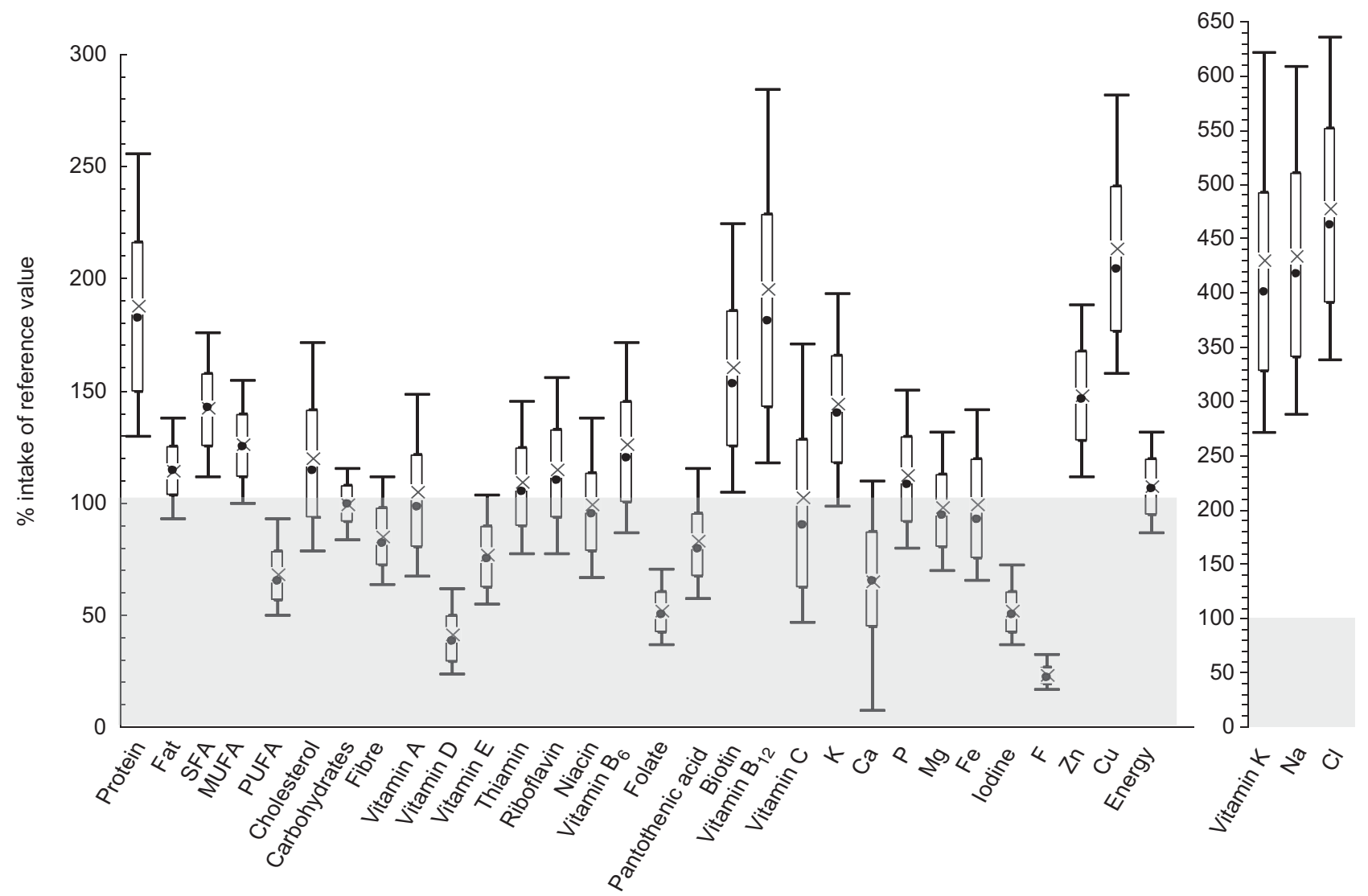

Fig. 1 Dietary nutrient and energy intakes in comparison with the D-A-CH reference values for nutrient intake among 1590 European adolescents aged 12.5-17.5 years from the Healthy Lifestyle in Europe by Nutrition in Adolescents (HELENA) Study. Data are presented as box-and-whisker plots in which the bottom and top of the box represent the 25th and 75th percentile (quartile 1 and quartile 3), respectively; the bottom and top of the whisker represent the 10th and 90th percentile, respectively; $\times$ represents the mean; and 0 represents the median; $\square$ represents intake inadequacy compared with the reference value

However, the intake of vitamin $\mathrm{D}$ was far below the acceptable range with only about $40 \%$ and $35 \%$ of the recommended intake in boys and girls, respectively. In contrast, vitamin $\mathrm{K}$ intake was about three to five times higher than the nutrient reference value.

Intakes of the water-soluble vitamins thiamin, riboflavin, niacin, vitamin $\mathrm{B}_{6}$, pantothenic acid, biotin, vitamin $\mathrm{B}_{12}$ and vitamin $\mathrm{C}$ met or exceeded the recommendations among the European adolescents. Only folate failed to reach approximately $50 \%$ of the recommended intake.

Regarding minerals, the intakes of $\mathrm{Na}$ and $\mathrm{Cl}$ were about three to five times higher than the recommended values in boys and girls among all age groups. Furthermore, the intakes of $\mathrm{K}, \mathrm{P}, \mathrm{Mg}, \mathrm{Zn}$ and $\mathrm{Cu}$ were sufficient. With respect to $\mathrm{Ca}$, boys aged 10-15 years had an acceptable intake of about $80 \%$ of the recommended minimum value, whereas the intake of the older boys was lower (about 70\%). For girls, Ca intake reached the lower acceptable level of about $75 \%$ of the recommendation in the youngest group, but only $55 \%$ of the recommended intake for the oldest age group. The intake of Fe was within the acceptable range among both genders, with only slightly lower values among girls of the youngest age groups. In contrast, iodine and $\mathrm{F}$ intakes were insufficient, as intakes did not reach more than about $55 \%$ of the recommendation for iodine and no more than $30 \%$ for $\mathrm{F}$.

Energy intakes of both genders were in line with the recommendations.

Finally, the weighted NQI derived from both absolute values and densities of the European adolescents, stratified by age group, are presented in Table 4 . The median NQI was approximately 72 in absolute value and 71 in terms of nutritional density (of a maximum value of 100). The NQI values were highest in 10- to 13-year-old boys (about 77 and 75 for absolute and density values, respectively) and lowest for 13- to 15-year-old girls (about 70 and 69 for absolute values and density values, respectively). Significant differences between boys and girls existed regarding the NQI density in the youngest age group $(10-<13$ years) and regarding both NQI absolute and NQI density in the middle age group (13-<15 years), with consistently higher values for boys. Since there is no specific cut-off for the NQI indicating an 'adequate' diet, the same cut-off as for evaluating single nutrient intake was used, i.e. $75 \%$. Thus, the overall nutritional quality was rather low among the European adolescents. 


\section{Discussion}

The present analysis provides for the first time detailed and comprehensive data from a standardized dietary assessment of the energy and nutrient intakes of adolescents across Europe. Compared with widely used nutrient reference intakes, the energy intake and the proportions of energy intake from carbohydrates, fat and protein are sufficient. However, the quality of fat intake needs to be improved in the European adolescents. For the micronutrients as well as for dietary fibre, the intake levels are mostly within an acceptable range, with the exceptions of vitamin $\mathrm{D}$, folate, iodine and $\mathrm{F}$ across the study population and $\mathrm{Ca}$ in older girls. However, regarding the nutritional quality as a whole, the European boys and girls have a rather low overall diet quality with a median NQI of about 72 and 71 for absolute and density values, respectively.

These results from the HELENA Study are generally concordant with earlier regional studies in several European countries. In comparison with the D-A-CH reference values (or with the similar US recommended daily allowance ${ }^{(48)}$ ), the protein intake of the HELENA adolescents was about twice as high. These findings are in line with the results of other European studies ${ }^{(22,49,50)}$. Although an increase in protein intake may have negative effects on bone health ${ }^{(51)}$, no long-term studies have revealed adverse effects of protein intake above the recommended amount ${ }^{(52)}$, i.e. at levels such as those in our sample. Moreover, in 6- to 18-yearold German ${ }^{(53)}$ and 17-year-old Danish ${ }^{(54)}$ children and adolescents, protein intake was positively associated with bone strength and mineralization. Thus, the described protein intake of the European adolescents might not be of concern.

The total intakes of fat and cholesterol in the HELENA sample are well in line with the guidelines. However, the proportions of energy from SFA and PUFA among the European adolescents are in need of improvement, since the intake of SFA exceeded the recommendations by about $40 \%$ and the intake of PUFA was found to be $40 \%$ lower. This pattern - which has also been described in other studies among European adolescents ${ }^{(49,55-58)}-$ may not only negatively influence present health but may also increase the risk for diabetes, dyslipidaemia or CVD later in life $\mathrm{e}^{(15,59)}$. Associations between fat intake during childhood and the quality of the adult diet two decades later have been reported ${ }^{(60)}$, and so it is of importance in the prevention of diet-related diseases to improve the dietary fat quality already in adolescence ${ }^{(61)}$.

Regarding micronutrients, most vitamins and minerals were consumed in accordance with the D-A-CH reference values used here. Some smaller deviations, e.g. a lower intake of vitamin $\mathrm{E}$ intake in younger boys, of Ca among older boys or of Fe in younger girls, might be attributed to the specific recommendations used here or to the skewed distribution of recommendations. Since the deviations do not exceed $30 \%$ and the recommended intakes are designed to be $20-30 \%$ higher than the average requirement, 
this might not be an indication of deficiencies ${ }^{(23)}$. Unfortunately, at the moment a diversity of nutrient reference values is used across Europe with considerable differences in the stated nutritional requirements. The nutritional quality of the diet of European adolescents based on nutrient intake would thus be estimated differently depending on what recommendations are used ${ }^{(62)}$. While some of the disparities can be attributed to physiological and environmental differences between populations, most are due to methodological differences ${ }^{(24)}$.

However, compared with the D-A-CH reference values, and also in comparison with the recommendations of most other European countries ${ }^{(24)}$, the intakes of vitamin D, folate, iodine and $\mathrm{F}$ were insufficient among European adolescents, as was the intake of $\mathrm{Ca}$ in 13- to 19-year-old girls. As shown in various publications ${ }^{(22,49,56)}$, vitamin D intake often reaches only about $40 \%$ of the recommended level. This is due to the fact that vitamin $\mathrm{D}$ is found naturally in only a few foods and even fortification which is a widely used technique to increase vitamin D intake in many industrialized countries - has only modest effects on vitamin D status ${ }^{(63)}$ and could not be considered in the HELENA Study. Of greater importance is the endogenous synthesis of vitamin D when the skin is exposed to sunlight. However, skin synthesis may not fully compensate for the low nutritional intake among all adolescents throughout Europe, because Europe is located over many degrees of latitude and skin production of vitamin D varies considerably at different latitudes ${ }^{(64)}$.

The intake of folate reached only about $50 \%$ of the recommendation in this sample. Other nutritional surveys describe intakes at a similar level ${ }^{(22,55)}$, with slightly higher values in Southern Europe ${ }^{(57)}$. Folate intake is difficult to assess because neither cooking losses nor food fortification were accounted for in HELENA-DIAT. Since folate deficiency is not only important for the prevention of neural tube defects in pregnancy but also of CVD and some malignancies ${ }^{(65)}$, the low folate intake among the European adolescents is of concern.

The intakes of iodine and $\mathrm{F}$ were also very low, reaching only about $50 \%$ and $20 \%$ of the recommendations, respectively. While the intake of $\mathrm{F}$ is not reported in other European surveys among adolescents, iodine intake has repeatedly been found to be below the recommended intakes ${ }^{(22)}$. Again these minerals are difficult to assess with HELENA-DIAT, since a major part of the iodine and $\mathrm{F}$ intake stems from fortification of e.g. table salt $^{(66,67)}$ and - in the case of $\mathrm{F}$ - swallowed toothpaste $^{(68)}$. However, deficiencies of these minerals can result in thyroid diseases ${ }^{(67)}$ and dental caries and should thus be prevented by comprehensive fortifications.

On the other hand, there are some micronutrients that were ingested in much higher amounts among the HELENA adolescents than recommended, i.e. vitamin K, $\mathrm{Na}$ and $\mathrm{Cl}$. However, the toxicity of vitamin $\mathrm{K}$ is extremely low and no upper level has been established ${ }^{(47)}$. The $\mathrm{Na}$ and $\mathrm{Cl}$ intakes equate to about $6 \mathrm{~g}$ of salt and thus around the accepted amount of daily salt intake ${ }^{(23)}$. However, this value is certainly highly underestimated since table salt is not considered in HELENA-DIAT and might be not completely assessed in processed or convenience food. Therefore, the actual salt intake - which includes table salt - might be critically high.

Furthermore, the median energy intake of adolescents participating in the HELENA study was about $11046 \mathrm{~kJ} / \mathrm{d}$ $(2640 \mathrm{kcal} / \mathrm{d})$ for boys and about $8619 \mathrm{~kJ} / \mathrm{d}(2060 \mathrm{kcal} / \mathrm{d})$ for girls and is therefore in the range presented in the European Nutrition and Health Report (9498$14518 \mathrm{~kJ} / \mathrm{d}(2270-3470 \mathrm{kcal} / \mathrm{d})$ for boys, $6820-9707 \mathrm{~kJ} / \mathrm{d}$ $(1630-2320 \mathrm{kcal} / \mathrm{d})$ for girls) ${ }^{(49)}$.

Nutritional indices are a way to assess overall diet quality with a single parameter ${ }^{(25)}$. However, most indices are calculated based on the arithmetical mean, which is known to balance data, i.e. deficiencies of a few nutrients are compensated for by adequate intakes of other nutrients ${ }^{(69-71)}$. Such a mathematical compensation is not, however, physiologically possible. The NQI is therefore based on the harmonic mean. In this, a low intake of one nutrient is weighted more heavily and decreases the overall nutritional quality disproportionately ${ }^{(26)}$. Compensation is thus reduced, corresponding more to metabolic pathways. The HELENA adolescents had a median absolute and density NQI of about 72 and 71, respectively. Unfortunately, the interpretation of these values is not straightforward, since specific cut-offs indicating an 'adequate' diet are not determined for most indices and are difficult to justify in the absence of an overall biomarker. Some studies have used the assumption that values below $67 \%$ of the optimum might indicate a suboptimal state of nutrition ${ }^{(69)}$, while others used the indices only to compare subsamples $^{(25,70,71)}$. We used the same cut-off as for single nutrients, i.e. $75 \%$, and found a rather low overall nutritional quality. Since the harmonic mean was used in this data analysis, the noticeable low intakes of folate, vitamin $\mathrm{D}$, iodine and $\mathrm{F}$ might have affected the NQI of the adolescents excessively. Under these considerations, the overall nutritional quality seems to be close to adequate among the HELENA sample.

Before giving any recommendation for supplementation of specific nutrients, the nutrient status of the European adolescents should be established ${ }^{(72)}$ and potential countryor population-specific situations should be taken into account. Some biomarker evaluations of nutritional status have already been executed or are in process in a HELENA sub-sample ${ }^{(73)}$.

Since the $24 \mathrm{~h}$ recall used in HELENA was identified to have substantial under-reporting bias ${ }^{(31,74)}, 214$ (12\%) under- and over-reporters were excluded from the analysis. The groups differed significantly in their adherence to the D-A-CH recommendations in nearly all nutrients (except for carbohydrates and fat) and in their NQI (data not shown). Furthermore, the included adolescents differed 
significantly from the rest of the whole HELENA sample regarding their anthropometric, socio-economic and health related characteristics (Table 1). These results point to the fact that data of the excluded adolescents were biased. Therefore, it can be assumed that the data obtained in the present analysis from the included participants only are more reliable and allow meaningful information about nutrient intake among European adolescents.

Some limitations of the study need to be mentioned. To begin with, the HELENA-CSS cohort is not a fully representative European sample, since due to the selection procedure of classes from all schools in the chosen cities representativeness can be at best achieved on the city level. However, this procedure was anticipated to give a fair approximation of the average situation, if the objective was to only describe the adolescents' characteristics, as was the case in the present study ${ }^{(27)}$. Furthermore, the $24 \mathrm{~h}$ recall used in the HELENA Study has been shown to be prone to under-reporting in a validation study ${ }^{(32,74)}$. Nevertheless, due to the exclusion of over- and underreporters in the present sample, systematic bias due to inadequate reporting should have been reduced. On the other hand, the exclusion might have induced selection bias since the misreporters might have a special food choice or eating behaviour. Furthermore, large withinperson variation might be of concern, given that dietary data were assessed only by two $24 \mathrm{~h}$ recalls. However, the large size of the sample (nearly 1600 adolescents) should have alleviated large within-person variations ${ }^{(75)}$. Additionally, sample weights that were constructed for the sample after over- and under-reporters were excluded calibrated the sample so that it matched the European population with regard to sex and age group. Another limitation is the fact that the $24 \mathrm{~h}$ recalls were all completed during school days (about the dietary intake of the previous day) and thus did not include information about the adolescents' diet on Fridays and Saturdays or on holidays. Moreover, a $24 \mathrm{~h}$ recall like HELENA-DIAT might not be an adequate tool to estimate some minerals like $\mathrm{Na}, \mathrm{Cl}$, iodine and $\mathrm{F}$ stemming mostly from (fortified) table salt, as well as the amount and quality of additional fat for meal preparation.

One of the major strengths of the present study, besides the large sample size, is the geographical spread over eight European cities. The sample consists of adolescents assessed using highly standardized and validated procedures; for example, all countries used the same $24 \mathrm{~h}$ recall (HELENA-DIAT) and the same food composition database (BLS). Another strength is the use of the Multiple Source Method taking into account both between- and withinindividual variability of the dietary intake data. Moreover, the current study is one of the first examining the overall nutrient intake and not just specific nutrients among adolescents at a pan-European level. We evaluated the overall nutritional quality of the adolescents with the NQI. Nutritional indices can be used to some extent to evaluate a diet's adequacy ${ }^{(71)}$. However, they are not appropriate to derive specific recommendations to improve nutritional quality, since the intake of single nutrients has to be considered. This has been conducted here extensively by the use of public health-oriented reference values ${ }^{(24)}$.

\section{Conclusion}

The present analysis of the HELENA Study provides new, reliable and comprehensive insight into the intake of a large number of nutrients among European adolescents. Compared with the D-A-CH reference values the intakes of most nutrients can be assumed to be adequate. However, further studies using suitable criteria to assess nutrient status are needed and further research should also investigate the high protein and salt intakes found in the HELENA adolescents.

Overall, public health initiatives should educate children and adolescents regarding balanced food choices (possibly with the addition of fortified foods or supplements for some critical nutrients, if evidence dictates), since health behaviour adopted in childhood and adolescence might be carried over into adulthood and may thus play an important role in the prevention of nutrition-related diseases.

\section{Acknowledgements}

Sources of funding: The HELENA Study took place with the financial support of the European Community Sixth RTD Framework Programme (Contract FOOD CT-2005007034). The contents of this paper reflect only the authors' views and the rest of HELENA Study Members and the European Community are not liable for any use that may be made of the information contained herein. Ethics: The ethical committee of each city approved the study. Conflicts of interest: The authors have no conflicts of interest. Authors' contributions: L.M. coordinated the project. L.M., M.G.-G. and M.K. designed and carried out the HELENA project. K.D. carried out the data analyses and drafted the manuscript. M.K. provided critical input on the data analyses and on earlier versions of the manuscript and supervised the study. I.H., S.D.H., Y.M., L.B., M.G.-G., C.L.D., M.C.-G., M.J.C., K.W. and E.P. contributed to interpretation of the data of the submitted manuscript. Each author has seen and approved the contents of the submitted manuscript. Acknowledgements: The participation of all adolescents in the HELENA Study is gratefully acknowledged.

\section{References}

1. Villa I, Yngve A, Poortvliet E et al. (2007) Dietary intake among under-, normal- and overweight 9- and 15-year-old Estonian and Swedish schoolchildren. Public Health Nutr 10, 311-322. 
2. Tucker LA, Seljaas GT \& Hager RL (1997) Body fat percentage of children varies according to their diet composition. I Am Diet Assoc 97, 981-986.

3. Guo SS, Wu W, Chumlea WC et al. (2002) Predicting overweight and obesity in adulthood from body mass index values in childhood and adolescence. Am J Clin Nutr 76, 653-658.

4. Amati L, Chiloiro M, Jirillo E et al. (2007) Early pathogenesis of atherosclerosis: the childhood obesity. Curr Pharm Des 13, 3696-3700.

5. Baker JL, Olsen LW \& Sorensen TI (2007) Childhood bodymass index and the risk of coronary heart disease in adulthood. $N$ Engl J Med 357, 2329-2337.

6. Must A, Spadano J, Coakley EH et al. (1999) The disease burden associated with overweight and obesity. JAMA 282, $1523-1529$

7. Law M (2000) Dietary fat and adult diseases and the implications for childhood nutrition: an epidemiologic approach. Am J Clin Nutr 72, 5 Suppl., 1291S-1296S.

8. Biro FM \& Wien M (2010) Childhood obesity and adult morbidities. Am J Clin Nutr 91, issue 5, 1499S-1505S.

9. Morrison JA, Glueck CJ, Daniels S et al. (2010) Determinants of persistent obesity and hyperinsulinemia in a biracial cohort: a 15-year prospective study of schoolgirls. $J$ Pediatr 157, 559-565.

10. Lustig RH (2006) Childhood obesity: behavioral aberration or biochemical drive? Reinterpreting the First Law of Thermodynamics. Nat Clin Pract Endocrinol Metab 2, 447-458.

11. Lytle LA \& Kubik MY (2003) Nutritional issues for adolescents Best Pract Res Clin Endocrinol Metab 17, 177-189.

12. van Lenthe FJ, Kemper CG \& van Mechelen W (1996) Rapid maturation in adolescence results in greater obesity in adulthood: the Amsterdam Growth and Health Study. Am J Clin Nutr 64, 18-24.

13. Dietz WH (1994) Critical periods in childhood for the development of obesity. Am J Clin Nutr 59, 955-959.

14. Mikkila V, Rasanen L, Raitakari OT et al. (2005) Consistent dietary patterns identified from childhood to adulthood: the Cardiovascular Risk in Young Finns Study. Br J Nutr 93, 923-931.

15. World Health Organization (2003) Diet, Nutrition, and the Prevention of Chronic Diseases. Joint WHO/FAO Expert Consultation. WHO Technical Report Series no. 916. Geneva: WHO.

16. Moreno LA (2008) Adolescence. In Nutrition in Practice, pp. 114-117 [B Koletzko, editor]. Basel: Karger.

17. Hoppu U, Lehtisalo J, Tapanainen H et al. (2010) Dietary habits and nutrient intake of Finnish adolescents. Public Health Nutr 13, 965-972.

18. Libuda L, Alexy U, Buyken AE et al. (2009) Consumption of sugar-sweetened beverages and its association with nutrient intakes and diet quality in German children and adolescents. Br J Nutr 101, 1549-1557.

19. Toselli S, Argnani L, Canducci E et al. (2010) Food habits and nutritional status of adolescents in Emilia-Romagna, Italy. Nutr Hosp 25, 613-621.

20. Royo-Bordonada MA, Gorgojo L, Martin-Moreno JM et al. (2003) Spanish children's diet: compliance with nutrient and food intake guidelines. Eur J Clin Nutr 57, 930-939.

21. Sette S, Le Donne C, Piccinelli R et al. (2011) The third Italian National Food Consumption Survey, INRAN-SCAI 2005-06 - part 1: nutrient intakes in Italy. Nutr Metab Cardiovasc Dis 21, 922-932.

22. Lambert J, Agostoni C, Elmadfa I et al. (2004) Dietary intake and nutritional status of children and adolescents in Europe. Br J Nutr 92, Suppl. 2, S147-S211.

23. German Nutrition Society, Austrian Nutrition Society, Swiss Society for Nutrition Research et al. (editors) (2000) Reference Values for Nutrient Intake ( $D-A-C H)$, 1 st ed. Frankfurt am Main: Umschau/Braus.
24. Prentice A, Branca F, Decsi T et al. (2004) Energy and nutrient dietary reference values for children in Europe: methodological approaches and current nutritional recommendations. Br J Nutr 92, Suppl. 2, S83-S146.

25. Thiele S, Mensink GB \& Beitz R (2004) Determinants of diet quality. Public Health Nutr 7, 29-37.

26. Gedrich K \& Karg G (2001) Dietary habits of German vs. Non-German residents in Germany. In Culinary Arts and Sciences III - Global and National Perspectives, pp. 419-428 [JSA Edwards, editor]. Al-Fayoum: Al-Karma Press.

27. Moreno LA, De Henauw S, Gonzalez-Gross M et al. (2008) Design and implementation of the Healthy Lifestyle in Europe by Nutrition in Adolescence Cross-Sectional Study. Int J Obes (Lond) 32, Suppl. 5, S4-S11.

28. Moreno LA, Gonzalez-Gross M, Kersting M et al. (2008) Assessing, understanding and modifying nutritional status, eating habits and physical activity in European adolescents: the HELENA (Healthy Lifestyle in Europe by Nutrition in Adolescence) Study. Public Health Nutr 11, 288-299.

29. Beghin L, Huybrechts I, Vicente-Rodriguez G et al. (2012) Main characteristics and participation rate of European adolescents included in the HELENA study. Arch Public Health 70, 14

30. Beghin L, Castera M, Manios Y et al. (2008) Quality assurance of ethical issues and regulatory aspects relating to good clinical practices in the HELENA Cross-Sectional Study. Int J Obes (Lond) 32, Suppl. 5, S12-S18.

31. Vereecken CA, Covents M, Matthys C et al. (2005) Young adolescents' nutrition assessment on computer (YANA-C). Eur J Clin Nutr 59, 658-667.

32. Vereecken CA, Covents M, Sichert-Hellert W et al. (2008) Development and evaluation of a self-administered computerized 24-h dietary recall method for adolescents in Europe. Int J Obes (Lond) 32, Suppl. 5, S26-S34.

33. Dehne LI, Klemm C, Henseler G et al. (1999) The German Food Code and Nutrient Data Base (BLS II.2). Eur $J$ Epidemiol 15, 355-359.

34. Haubrock J, Nothlings U, Volatier JL et al. (2011) Estimating usual food intake distributions by using the multiple source method in the EPIC-Potsdam Calibration Study. J Nutr $\mathbf{1 4 1}$, 914-920.

35. Schofield WN (1985) Predicting basal metabolic rate, new standards and review of previous work. Hum Nutr Clin Nutr 39, Suppl. 1, 5-41.

36. Goldberg GR, Black AE, Jebb SA et al. (1991) Critical evaluation of energy intake data using fundamental principles of energy physiology: 1 . Derivation of cut-off limits to identify under-recording. Eur J Clin Nutr 45, 569-581.

37. Nagy E, Vicente-Rodriguez G, Manios Y et al. (2008) Harmonization process and reliability assessment of anthropometric measurements in a multicenter study in adolescents. Int J Obes (Lond) 32, Suppl. 5, S58-S65.

38. Cole TJ, Bellizzi MC, Flegal KM et al. (2000) Establishing a standard definition for child overweight and obesity worldwide: international survey. BMJ 320, 1240-1243.

39. Slaughter MH, Lohman TG, Boileau RA et al. (1988) Skinfold equations for estimation of body fatness in children and youth. Hum Biol 60, 709-723.

40. Iliescu C, Beghin L, Maes L et al. (2008) Socioeconomic questionnaire and clinical assessment in the HELENA Cross-Sectional Study: methodology. Int $J$ Obes (Lond) 32, Suppl. 5, S19-S25.

41. United Nations Educational, Scientific and Cultural Organization (2006) International Standard Classification of Education ISCED 1997 (May 2006, Re-edition). http:// www.uis.unesco.org/Library/Documents/isced97-en.pdf

42. Currie C, Molcho M, Boyce W et al. (2008) Researching health inequalities in adolescents: the development of the Health Behaviour in School-Aged Children (HBSC) family affluence scale. Soc Sci Med 66, 1429-1436. 
43. Hagstromer M, Bergman P, De Bourdeaudhuij I et al. (2008) Concurrent validity of a modified version of the International Physical Activity Questionnaire (IPAQ-A) in European adolescents: The HELENA Study. Int J Obes (Lond) 32, Suppl. 5, S42-S48.

44. Ottevaere C, Huybrechts I, De Bourdeaudhuij I et al. (2011) Comparison of the IPAQ-A and Actigraph in relation to VO2max among European adolescents: the HELENA study. J Sci Med Sport 14, 317-324.

45. IPAQ Group (2005) Guidelines for Data Processing and Analysis of the International Physical Activity Questionnaire (IPAQ) - Short and Long Forms. http://www.ipaq. ki.se/scoring.pdf

46. Kersting M, Sichert-Hellert W, Vereecken CA et al. (2008) Food and nutrient intake, nutritional knowledge and dietrelated attitudes in European adolescents. Int J Obes (Lond) 32, Suppl. 5, S35-S41.

47. Institute of Medicine (2001) Dietary Reference Intakes for Vitamin A, Vitamin K, Arsenic, Boron, Chromium, Copper, Iodine, Iron, Manganese, Molybdenum, Nickel, Silicon, Vanadium and Zinc. Washington, DC: National Academy Press.

48. National Academy of Sciences, Institute of Medicine, Food and Nutrition Board (2005) Dietary Reference Intakes for Energy, Carbohydrate, Fiber, Fat, Fatty Acids, Cholesterol, Protein and Amino Acids. Washington, DC: National Academy Press.

49. Elmadfa I, Meyer A, Nowak V et al. (2009) European nutrition and health report 2009. Forum Nutr 62, 1-405.

50. Parizkova J (2000) Dietary habits and nutritional status in adolescents in Central and Eastern Europe. Eur J Clin Nutr 54, Suppl. 1, S36-S40.

51. Barzel US \& Massey LK (1998) Excess dietary protein can adversely affect bone. J Nutr 128, 1051-1053.

52. Ginty F (2003) Dietary protein and bone health. Proc Nutr Soc 62, 867-876.

53. Alexy U, Remer T, Manz F et al. (2005) Long-term protein intake and dietary potential renal acid load are associated with bone modeling and remodeling at the proximal radius in healthy children. Am J Clin Nutr 82, 1107-1114.

54. Budek AZ, Hoppe C, Ingstrup H et al. (2007) Dietary protein intake and bone mineral content in adolescents The Copenhagen Cohort Study. Osteoporos Int 18, $1661-1667$

55. Elmadfa I \& Freisling H (2009) Nutritional status in Europe: methods and results. Nutr Rev 67, Suppl. 1, S130-S134.

56. Samuelson G (2000) Dietary habits and nutritional status in adolescents over Europe. An overview of current studies in the Nordic countries. Eur J Clin Nutr 54, Suppl. 1, S21-S28.

57. Cruz JA (2000) Dietary habits and nutritional status in adolescents over Europe - Southern Europe. Eur J Clin Nutr 54, Suppl. 1, S29-S35.

58. Rolland-Cachera MF, Bellisle F \& Deheeger M (2000) Nutritional status and food intake in adolescents living in Western Europe. Eur J Clin Nutr 54, Suppl. 1, S41-S46.
59. German Nutrition Society (2006) Evidence Based Guidelines: Fat Consumption and Certain Nutrition-Related Diseases. Bonn: German Nutrition Society.

60. Mikkila V, Rasanen L, Raitakari OT et al. (2004) Longitudinal changes in diet from childhood into adulthood with respect to risk of cardiovascular diseases: the Cardiovascular Risk in Young Finns Study. Eur J Clin Nutr 58, 1038-1045.

61. Spinneker A, Egert S, Gonzalez-Gross M et al. (2012) Lipid, lipoprotein and apolipoprotein profiles in European adolescents and its associations with gender, biological maturity and body fat - The HELENA Study. Eur J Clin Nutr 66, 727-735.

62. Tabacchi G, Wijnhoven TM, Branca F et al. (2009) How is the adequacy of micronutrient intake assessed across Europe? A systematic literature review. Br J Nutr 101, Suppl. 2, S29-S36.

63. Ovesen L, Andersen R \& Jakobsen J (2003) Geographical differences in vitamin D status, with particular reference to European countries. Proc Nutr Soc 62, 813-821.

64. Kimlin MG, Downs NJ \& Parisi AV (2003) Comparison of human facial UV exposure at high and low latitudes and the potential impact on dermal vitamin D production. Photochem Photobiol Sci 2, 370-375.

65. Molloy AM (2002) Folate bioavailability and health. Int J Vitam Nutr Res 72, 46-52.

66. World Health Organization (1995) Fluorides and oral health. Bull World Health Organ 73, 124-125.

67. Delange F (2002) Iodine deficiency in Europe and its consequences: an update. Eur J Nucl Med Mol Imaging 29, Suppl. 2, S404-S416.

68. Oganessian E, Ivancakova R, Lencova E et al. (2011) Alimentary fluoride intake in preschool children. $B M C$ Public Health 11, 768.

69. Madden J \& Yoder MD (1972) Program evaluation: food stamps and commodity distribution in rural areas of central Pennsylvania. Pennsylvania Agricultural Experiment Station Bulletin 78, 1-119.

70. Basiotis P, Guthrie J, Bowman S et al. (1995) Construction and evaluation of a diet status index. Fam Econ Nutr Rev 8, 2-13.

71. Waijers PM, Feskens EJ \& Ocke MC (2007) A critical review of predefined diet quality scores. Br J Nutr 97, 219-231.

72. Al-Tahan J, Gonzalez-Gross M \& Pietrzik K (2006) B-vitamin status and intake in European adolescents. A review of the literature. Nutr Hosp 21, 452-465.

73. Gonzalez-Gross M, Valtuena J, Breidenassel C et al. (2012) Vitamin D status among adolescents in Europe: the Healthy Lifestyle in Europe by Nutrition in Adolescence study. Br J Nutr 107, 755-764.

74. Vereecken C, Dohogne S, Covents M et al. (2010) How accurate are adolescents in portion-size estimation using the computer tool Young Adolescents' Nutrition Assessment on Computer (YANA-C)? Br J Nutr 103, 1844-1850.

75. van 't Veer P (1994) Measuring nutritional exposures including biomarkers. Proc Nutr Soc 53, 27-35. 